\title{
Application of seventeen two-locus models in genome-wide association studies by two-stage strategy Adan Niu ${ }^{1}$, Zhaogong Zhang ${ }^{1,2}$ and Qiuying Sha*1
}

\author{
Addresses: ${ }^{1}$ Department of Mathematical Sciences, Michigan Technological University, Houghton, Michigan 49931, USA and \\ ${ }^{2}$ School of Computer Science and Technology, Heilongjiang University, Harbin 150080, PR China \\ E-mail: Adan Niu - aniu@mtu.edu; Zhaogong Zhang - zhaogong@mtu.edu; Qiuying Sha* - qsha@mtu.edu \\ ${ }^{*}$ Corresponding author
}

from Genetic Analysis Workshop 16

St Louis, MO, USA 17-20 September 2009

Published: 15 December 2009

BMC Proceedings 2009, 3(Suppl 7):S26 doi: 10.1186/1753-656I-3-S7-S26

This article is available from: http://www.biomedcentral.com/I753-656I/3/S7/S26

(c) 2009 Niu et al; licensee BioMed Central Ltd.

This is an open access article distributed under the terms of the Creative Commons Attribution License (http://creativecommons.org/licenses/by/2.0), which permits unrestricted use, distribution, and reproduction in any medium, provided the original work is properly cited.

\begin{abstract}
The goal of this paper is to search for two-locus combinations that are jointly associated with rheumatoid arthritis using the data set of Genetic Analysis Workshop 16 Problem I. We use a twostage strategy to reduce the computational burden associated with performing an exhaustive twolocus search across the genome. In the first stage, the full set of 531,689 single-nucleotide polymorphisms was screened using univariate testing. In the second stage, all pairs made from the 500 single-nucleotide polymorphisms with the lowest $p$-values from the first stage were evaluated under each of 17 two-locus models. Our analyses identified a two-locus combination - rs6939589 and rs I 1634386 - that proved to be significantly associated with rheumatoid arthritis under a Rec $x$ Rec model ( $p$-value $=0.045$ after adjusting for multiple tests and multiple models).
\end{abstract}

\section{Background}

Although the primary interest of genome-wide association studies (GWAS) is to identify single-nucleotide polymorphisms (SNPs) with detectable marginal effects, it is also of interest to identify SNPs that have an interaction effect. Testing all possible pairs of loci to identify interactions creates many practical difficulties. In this article, we use a two-stage strategy to search for two-locus joint effects, which effectively reduces the computation time. In the first stage, we only selected loci that met an initial threshold. In the second stage, any locus that met the first-stage threshold was tested under each of 17 two-locus joint effect models. By applying this two-stage analysis to Genetic Analysis Workshop 16
(GAW16) Problem 1, we successfully identified two SNPs that are jointly associated with rheumatoid arthritis (RA) and could not be identified by singlelocus analysis.

\section{Methods}

Two-locus analysis based on 17 two-locus models

In this article, we propose 17 two-locus models, which include 8 epistatic models and 9 multiplicative models (Figure 1).

The 17 two-locus models can be described by the following log linear model $\log P($ Disease $\mid g)=\beta_{0}+\beta_{1} X$, where $g$ is the two-locus genotype; for the eight epistatic 


\section{(a) Epistatic models}

\begin{tabular}{|c|c|c|c|}
\hline \multicolumn{4}{|c|}{ Dom $\cap$ Dom } \\
\hline $\mathrm{b}$ & $a$ & $a$ & $a$ \\
\hline $\mathrm{bB}$ & $a$ & $\beta$ & $\beta$ \\
\hline $\mathrm{B}$ & $a$ & $\beta$ & $\beta$ \\
\hline
\end{tabular}
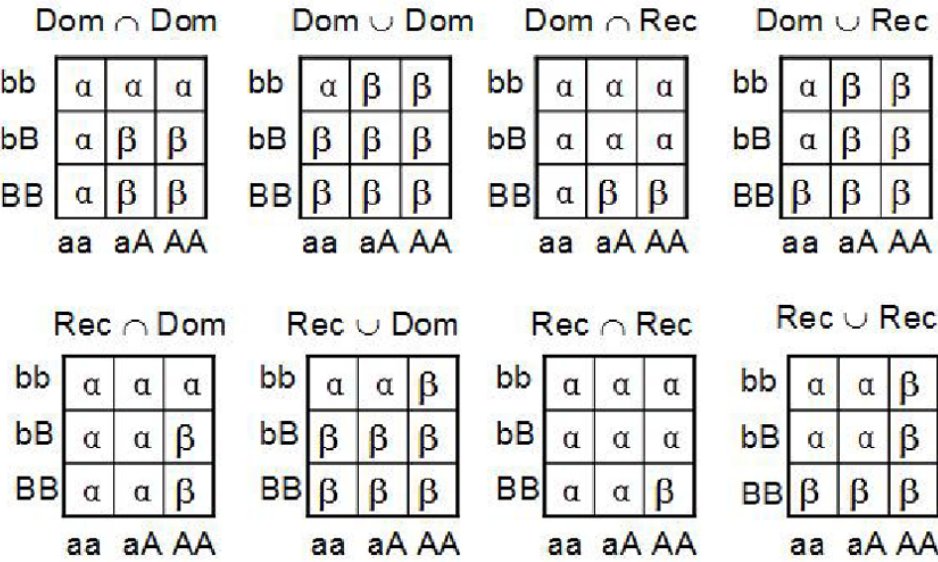

$\operatorname{Rec} \cup \operatorname{Rec}$

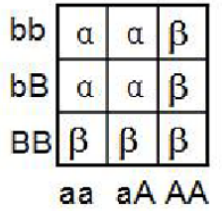

\section{(b) Multiplicative models}

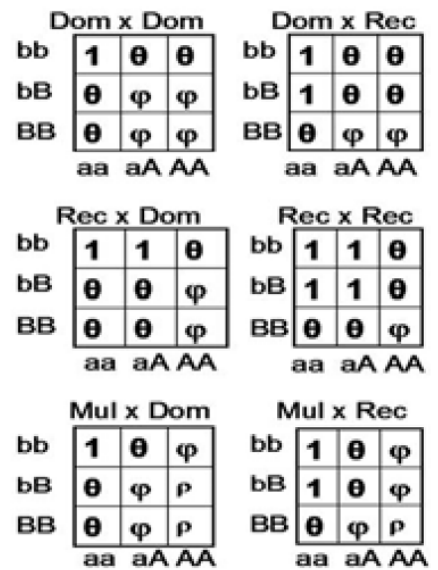

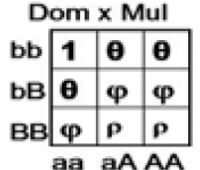

Rec x Mul bb \begin{tabular}{|l|l|l|}
\hline 1 & 1 & $\theta$ \\
\hline
\end{tabular} \begin{tabular}{l|l|l|l|}
\hline $\boldsymbol{\theta}$ & $\boldsymbol{\theta}$ & $\varphi$ \\
\hline
\end{tabular}

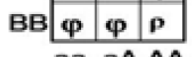
aa aA AA

Mul x Mul

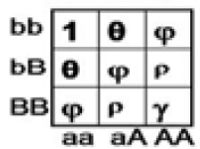

Figure I

Seventeen two-locus models. $\mathrm{A}$ and $\mathrm{B}$ are the high-risk alleles in the two markers. For the epistatic models, $\alpha$ and $\beta$ are the penetrances. For the multiplicative models, the symbol in each cell denotes the relative risk of this cell. $\phi=\theta^{2}, \rho=\theta^{3}$, and $\gamma=\theta^{4}$.

models in which the nine two-locus genotypes can be divided into high-risk group and low-risk group, $X=1$ if the genotype $g$ belongs to the high-risk group and $X=0$ otherwise; for the nine multiplicative models, $X=x_{1}+x_{2}$, where $x_{1}$ is the dominant, recessive, or additive coding of the genotype at the first SNP for a dominant, recessive, or multiplicative model, respectively, and $x_{2}$ is similarly defined for the second SNP. For the $i^{\text {th }}$ individual, let $y_{i}$ denote the trait value ( 1 for diseased individual and 0 for normal individual) and $X_{i}$ denote the numerical code of the genotype ( $X$ in the log linear model). The score test statistic is given by

$$
T_{\text {score }}=\frac{\left(\sum_{i=1}^{N}\left(X_{i}-\bar{X}\right)\left(y_{i}-\bar{y}\right)\right)^{2}}{\bar{y}(1-\bar{y}) \sum_{i=1}^{N}\left(X_{i}-\bar{X}\right)^{2}},
$$

where $N$ is the sample size, $\bar{X}$ is the average of $X_{1}, \ldots, X_{N}$ and $\bar{y}$ is the mean of $y_{1}, \ldots, y_{N}$. Under null hypothesis of no association, $T_{\text {score }}$ asymptotically follows a chi-square distribution with one degree of freedom (df). Though we use a unified test statistic $T_{\text {score }}$ for all of the models, the 17 models correspond to 17 different tests. Different models correspond to different genotype coding, and thus different values of $X_{i}$ in $T_{\text {score }}$. For example, under Rec $\cap$ Rec epistatic model, $X=1$ if the two-locus genotype is AABB; $X=0$ otherwise; under $\operatorname{Rec} \times \operatorname{Rec}$ multiplicative model, $X=x_{1}+x_{2}$, where $x_{1}=1$ if the genotype at the first SNP is AA and $x_{1}=0$ otherwise; $x_{2}$ is similarly defined for the second SNP.
The method to search for significant two-locus combinations for each of the 17 models has the following two steps. In the first step, three one-df chi-square tests (corresponding to dominant, recessive, and additive models) are used to test for association at each SNP. For a SNP, let $P$ denote the smallest $p$-value of the three tests. We select $M$ SNPs with the smallest $P(M=500$ was used in this study). In the second step, under each of the 17 two-locus models, we apply a two-locus association test $T_{\text {score }}$ to each of the $L$ two-locus combinations among the $M$ selected SNPs, where $L=M(M-1) / 2$. In this step, we get a $p$-value (called raw $p$-value) for each of the $L$ two-locus combinations and each of the 17 two-locus models.

Let $p_{i j}$ denote the raw $p$-value of the $l^{\text {th }}$ two-locus combination under the $j^{\text {th }}$ two-locus model. A permutation procedure is then used to adjust for multiple tests and multiple models. For each permutation, we randomly shuffle the case and control status. Based on the permuted data, we redo the single-marker test for each SNP, select M SNPs with the smallest $p$-values, and then test each of the two-locus combinations among the $M$ selected SNPs under each of the 17 models. For the $k^{\text {th }}$ permutation, let $p_{l j}^{k}$ denote the $p$-value of the two-locus test for the $l^{\text {th }}$ two-locus combinations under the $j^{\text {th }}$ model and let $p_{\min }^{k}=\min _{1 \leq l \leq L, 1 \leq j \leq 17}\left\{p_{l j}^{k}\right\}$. Suppose that we perform the permutation $K$ times (we use $K=1000$ in this study). Then, the overall $p$-value of the two-locus test for the $l^{\text {th }}$ two-locus combination under the $j^{\text {th }}$ model is given by $P_{l j}=\frac{\#\left\{k: p_{\min }^{k}<p_{l j}\right\}}{K}$. In this way, we can adjust 
for multiple testing for $L$ two-locus tests and 17 models and we also can adjust for the fact that the M SNPs to do two-locus analysis were chosen as the "top" signals from a much larger set.

\section{Control for population stratification}

The two-locus analysis discussed in the last section is valid under the assumption that all sampled individuals come from a homogeneous population. Thus, we first check this assumption in the data set of GAW16 Problem 1 . For this purpose, we randomly choose 1000 SNPs and perform association test at each of the 1000 SNPs using the one-df chi-square test. The histogram of the $p$-values of the 1000 tests is given in Figure 2a. If all sampled individuals come from a homogeneous population, the $1000 p$-values should follow a uniform distribution. Figure 2a shows that the data set of GAW16 Problem 1 has the problem of population stratification. To control for population stratification, we propose to use the following two methods:

1. Genome Control (GC) method: the GC method $[1,2]$ rescales the chi-square test statistic. Suppose that we have $N$ one-df chi-square tests (single-marker test or two-locus test) with test statistics $T_{1}, T_{2}, \ldots, T_{N}$. The GC method rescales $T_{i}$ as $S_{i}=\frac{T_{i}}{b}(i=1,2, \ldots, N)$, where $b=$ median $\left\{T_{1}, T_{2}, \ldots, T_{N}\right\} / 0.465$ and considers $S_{i}$ following a chi-square distribution with one $\mathrm{df}$.

2. Permutation GC method: Suppose that we have $N$ one-df chi-square tests and rescale the test statistics as $S_{1}, S_{2}, \ldots, S_{N}$ by the GC method. Then, we carry out permutation $K$ times. For each permutation, we perform $N$ chi-square tests based on the permuted data and rescale the test statistics by GC method. For the $k^{\text {th }}$ permutation, let $T_{1}^{k}, T_{2}^{k}, \ldots, T_{N}^{k}$ denote the chisquare test statistics. We rescale them as $S_{1}^{k}, S_{2}^{k}, \ldots, S_{N}^{k}$ by GC method. Then, the $p$-value of the $i^{\text {th }}$ test is given by $p_{i}=\#\left\{k: S_{i}^{k}>S_{i}\right\} / K$.

To test whether the two methods can control for population stratification, we randomly chose 1000 SNPs from the data set of GAW16 Problem 1. The GC and permutation GC methods were used to test association for the 1000 SNPs. The histograms of the $p$-values from the two methods are shown in Figure 2, b and c. These two panels show that the distributions of the $p$-values are very similar to a uniform distribution, which indicates that both the GC and permutation GC methods can control for population stratification, at least for the data set of GAW16 Problem 1.

\section{Results}

The GAW16 Problem 1 data set contains genotypes at 531,690 SNPs on chromosomes 1-22 (868 cases and 1194 controls). We first performed single-marker analysis. Three one-df chi-square tests (corresponding to dominant, recessive, and additive models) rescaled by GC method were applied to each SNP. The smallest $p$-value of the three tests at each SNP was adjusted by Bonferroni correction for the three models and all the SNPs. From the single-marker analysis, we found one SNP (rs2476601) on chromosome 1 and 183 SNPs on chromosome 6 with adjusted $p$-values less than 0.05 after Bonferroni correction. The 183 SNPs that we found on chromosome 6 are in a region (29930240 to $33149012 \mathrm{bp}$ ) in high linkage disequilibrium with HLA-DRB1, a factor known to have a strong association with RA [3-6]. The SNP (rs2476601) on chromosome 1 is in the hematopoietic-specific protein tyrosine phosphatase gene, PTPN22, which has been identified to be associated with RA $[6,7]$. (a) Chi-square test

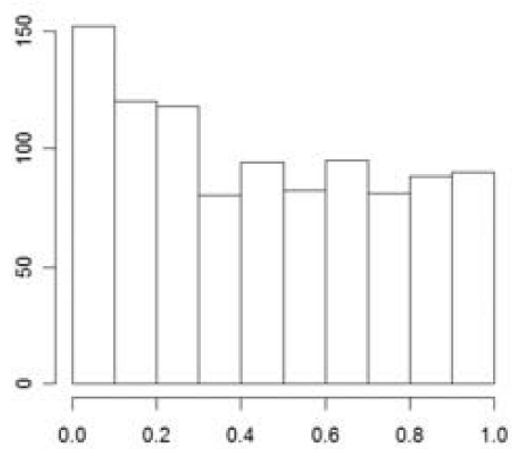

(b) GC method

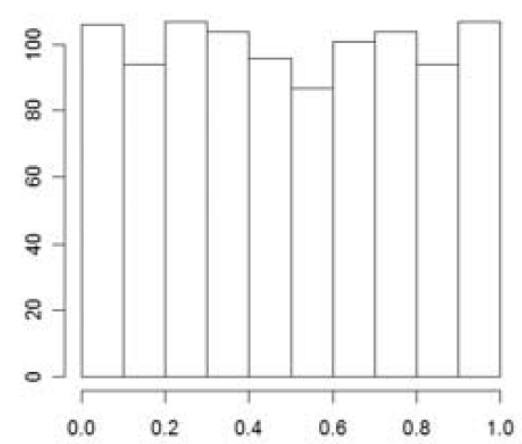

(c) Permutation GC method

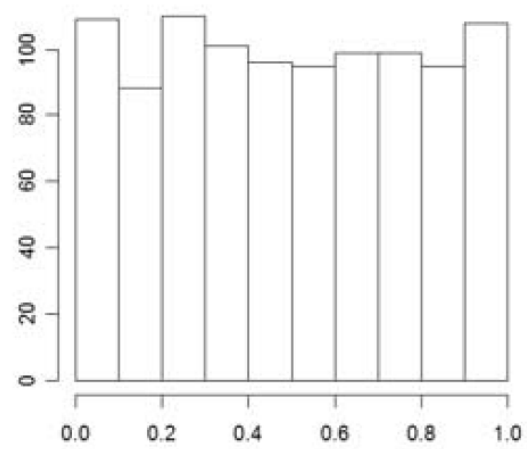

Figure 2

Histograms of the $p$-values of the three tests. 
We used the permutation GC method with 1000 permutations to carry out the two-locus analysis. In the analysis, we excluded SNPs within $10 \mathrm{Mbp}$ of any of the SNPs surviving Bonferroni correction from the singlemarker analysis. Then, we performed the two-stage, twolocus analysis among the remaining SNPs. For each pair of SNPs, individuals with missing genotypes at either locus were not included in that analysis. After adjusting for multiple tests and multiple SNPs, we found one twolocus combination - rs6939589 on chromosome 6 and rs11634386 on chromosome 15 - significantly associated with RA. The two-locus combination followed the Rec $x$ Rec multiplicative model with a $p$-value of 0.045 .

\section{Discussion}

Although there is growing appreciation that searching for epistatic interactions in humans may be a fruitful endeavor, there are still a number of practical difficulties associated with testing all possible pair-wise comparisons in the case of GWAS, such as data storage requirements, computation time, and multiple testing. In this report we used a computationally efficient twostage analysis to search for joint effects of genes for GAW16 Problem 1. Applying this strategy to GAW16 Problem 1, we have successfully identified two loci that are significantly associated with RA. These two loci would not have been identified as significant by singlemarker analysis after correction for multiple tests.

Computational efficiency has imposed limits on our two-stage strategy. Because our approach only searches two-locus interaction among the top M SNPs according to the marginal effects, the loci with strong interaction effects but weak marginal effects may not be detected. Furthermore, our approach only considers 17 interaction patterns (corresponding to the 17 two-locus models). The proposed two-stage strategy may have low power to detect interactions whose patterns depart from the 17 two-locus models.

\section{Conclusion}

Our two-locus analysis showed that a two-locus combination, rs6939589 and rs11634386, is significantly associated with RA.

\section{List of abbreviations used}

GAW16: Genetic Analysis Workshop 16; GC: Genome control; GWAS: Genome-wide association study; RA: Rheumatoid arthritis; SNP: Single-nucleotide polymorphism.

\section{Competing interests}

The authors declare that they have no competing interests.

\section{Authors' contributions}

AN performed the statistical analysis and wrote the first draft of the manuscript. $\mathrm{ZZ}$ participated in the study design and helped to draft the manuscript. QS contributed to the design of the study and to the manuscript preparation. All authors read and approved the final manuscript.

\section{Acknowledgements}

The Genetic Analysis Workshops are supported by $\mathrm{NIH}$ grant ROI GM03 I575 from the National Institute of General Medical Sciences. This work was supported by NIH grants ROI GM069940 and the OverseasReturned Scholars Foundation of Department of Education of Heilongjiang Province (II52HZOI).

This article has been published as part of BMC Proceedings Volume 3 Supplement 7, 2009: Genetic Analysis Workshop 16. The full contents of the supplement are available online at http://www.biomedcentral.com/ $1753-656 \mid / 3$ ? issue $=$ S7.

\section{References}

I. Devlin B and Roeder K: Genomic control for association studies. Biometrics 1999, 55:997-1004.

2. Devlin B, Roeder K and Wasserman L: Genomic control, a new approach to genetic-based association studies. Theor Pop Biol 200I, 60:155-166.

3. du Montcel ST, Michou L, Petit-Teixeira E, Osorio J, Lemaire I Lasbleiz S, Pierlot C, Quillet P, Bardin T, Prum B, Cornelis F and Clerget-Darpoux F: New classification of HLA-DRBI alleles supports the shared epitope hypothesis of rheumatoid arthritis susceptibility. Arthritis Rheum 2005, 52:1063-1068.

4. Stastny P: Mixed lymphocyte cultures in the rheumatoid arthritis. J Chin Invest 1976, 57: I |48-1 I57.

5. Stastny P: Association of the B-cell alloantigen DRw4 with rheumatoid arthritis. N EnglJ Med 1978, 298:869-87I.

6. Bowes J and Barton A: Recent advances in the genetics of RA susceptibility. Rheumatology 2008, 47:399-402.

7. Carlton $\mathrm{V}, \mathrm{Hu} \mathrm{X}$ and Chokkalingam A: PTPN22 genetic variation: evidence for multiple variants associated with rheumatoid arthritis. Am J Hum Genet 2005, 77:567-581.

\footnotetext{
Publish with Biomed Central and every scientist can read your work free of charge

"BioMed Central will be the most significant development for disseminating the results of biomedical research in our lifetime."

Sir Paul Nurse, Cancer Research UK

Your research papers will be:

- available free of charge to the entire biomedical community

- peer reviewed and published immediately upon acceptance

- cited in PubMed and archived on PubMed Central

- yours - you keep the copyright 